\title{
Unusual cause of paraplegia in a child of 5 years
}

\author{
Authors: \\ Nausheen Khan ${ }^{1}$ \\ Irma Vandewerke \\ Affiliations: \\ ${ }^{1}$ Department of Radiology, \\ Kalafong Hospital and \\ University of Pretoria, \\ South Africa \\ Correspondence to: \\ Nausheen Khan \\ Email: \\ nausheenkhan5@yahoo.com \\ Postal address: \\ Private Bag X396, Pretoria \\ 0001, South Africa \\ Dates: \\ Received: 15 Nov. 2013 \\ Accepted: 05 Jan. 2014 \\ Published: 04 July 2014 \\ How to cite this article: \\ Khan N, Vandewerke I. \\ Unusual cause of paraplegia \\ in a child of 5 years. S Afr J \\ Rad. 2014;18(1); Art. \#594, \\ 3 pages. http://dx.doi. \\ org/10.4102/sajr.v18i1.594

\section{Copyright:} \\ (C) 2014. The Authors. \\ Licensee: AOSIS \\ OpenJournals. This work \\ is licensed under the \\ Creative Commons \\ Attribution License.
}

Read online:
'Hydatid' originates from the Greek word meaning 'watery vesicle'. It refers to a cyst formed as a result of infestation by larvae of the tapeworm Echinococcus granulosus, endemic to sheepraising areas of the world. Humans are an accidental intermediary host, with lungs and liver most commonly affected. Hydatid involvement of the spine accounts for less than $1 \%$ of the total cases of hydatid disease and isolated extradural involvement is even rarer. We report a case of extradural hydatid cyst involving a boy of 5 years.

\section{Case report}

A 5-year-old boy accompanied by his grandmother presented with the inability to walk for five months. On examination he was paraplegic with bedsores, increased tone, brisk reflex and clonus of the lower limbs. There were contractures of both ankles and he had no bladder or bowel control. Blood count and chemistry were normal. A serology for amoeba and cysticercosis was done that suggested past exposure to Entamoeba histolytica. Echinococcus serology was not done.

Radiography of the chest did not reveal any abnormality. Radiography of the thoracolumbar spine demonstrated a levoscoliosis with the apex at T7 and flattening of the pedicles from T5 to T7 suggestive of an intraspinal mass (Figure 1). Computed tomography (CT) of the same area showed a widened canal with a well circumscribed oval (dome-shaped) extradural cystic mass measuring $4.8 \mathrm{~cm} \times 2 \mathrm{~cm} \times 1.6 \mathrm{~cm}$. The cyst extended through the left T6/7 intervertebral foramen (Figure 2). There was pressure erosion of the left $\mathrm{T} 6$ transverse process and severe pressure effect and compression on the spinal cord with anterior displacement. A differential diagnosis of cystic neurofibroma and hydatid cyst was considered. The patient was referred for magnetic resonance imaging (MRI), which confirmed an extradural lobulated cystic mass extending from T4 to T7 and extending through the left T6/7 widened neural foramen (Figure 3). There was no peripheral rim enhancement on intravenous injection of gadolinium (Figure 4). The lesion occupied the entire spinal canal with compression of the spinal cord from T4 to T8. A cystic mass was also demonstrated in segment 4 of the liver with similar signal intensity as the spinal lesion (Figure 5). These features were highly suggestive of an Echinococcus cyst, with differential diagnosis of cysticercosis, cystic neoplasm such as schwannoma, and arachnoid cyst.

The patient underwent surgery with laminectomy and removal of the cyst which contained clear contents. The surgical appearance was in keeping with that of a hydatid cyst. The cyst was removed and the specimen sent for histology, which revealed an open cyst with a relatively thin wall and a laminated appearance with dense eosinophilic granular debris on the surface, features highly suggestive of a hydatid cyst.

\section{Discussion}

Hydatid disease is a rare infection caused by the larvae of Taenia, Echinococcus granulosus, and less commonly Echinococcus multilocularis. According to Islekel et al., cited by Arif et al., ${ }^{1}$ bone disease was first described by Bidloo in 1708. The first description of spinal hydatid disease was made by Churrier in 1807, and the first surgical intervention was reported by Reydellet in 1819.1,2

Hydatid disease of the spine affects the vertebral column in $0.2 \%-1 \%$ of all patients. ${ }^{3}$ Due to the rarity of its presentation, clinicians should include spinal hydatid disease in the differential list for cystic masses of the spine causing paralysis; otherwise this potentially curable disease may be missed, or a delay in diagnosis may occur, as did in our case.

The disease presents with radiculopathy, myelopathy and local pain if the bone is destroyed, and signs of cord compression. In some patients the spinal hydatid cyst has the potential to grow to enormous size. It can clinically remain asymptomatic for years until it becomes large enough to produce symptoms. Cyst rupture can lead to severe anaphylaxis and death.,7 


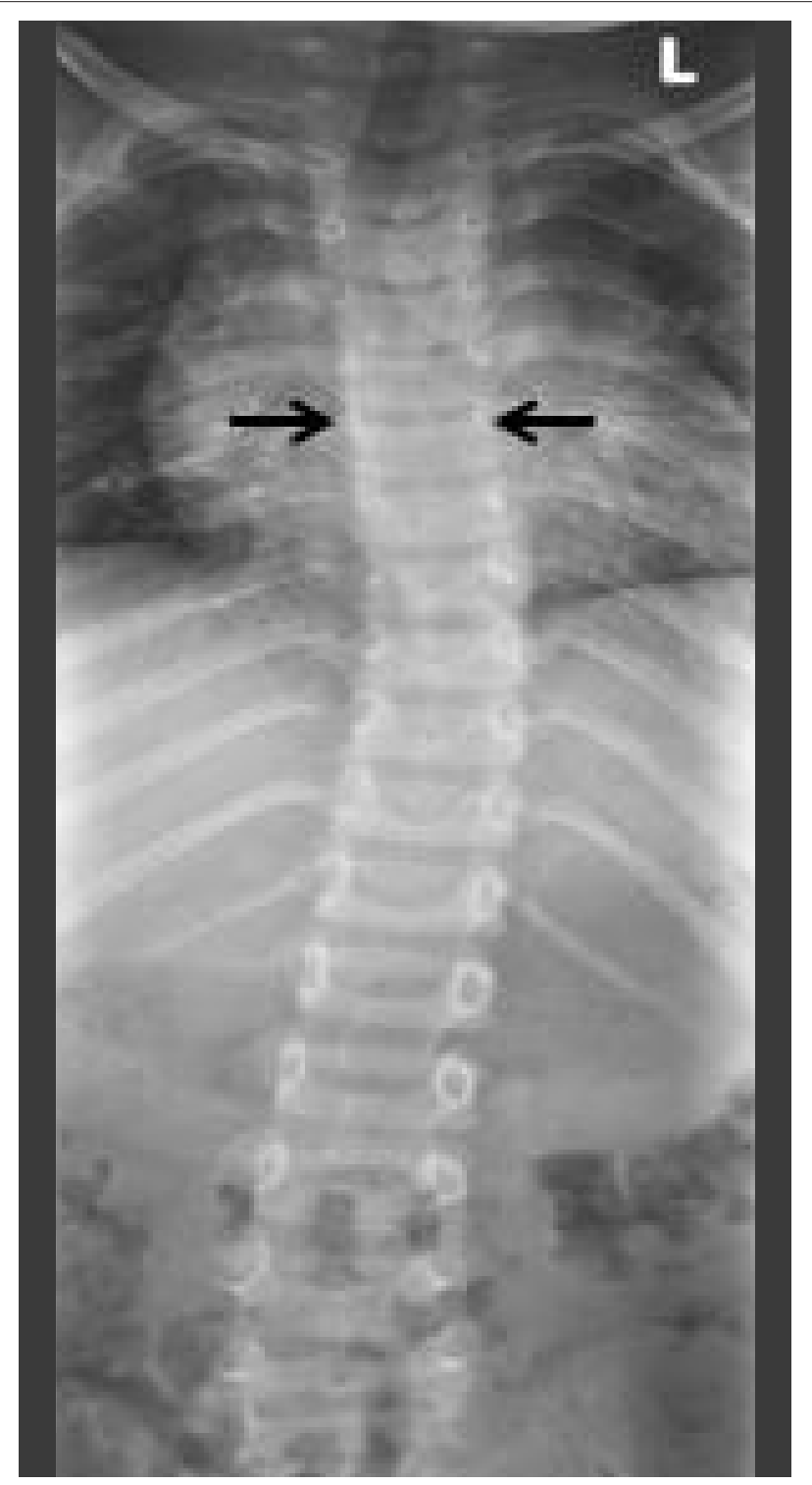

FIGURE 1: Anterior posterior view of the thoracolumbar spine shows levoscoliosis with apex at T7 and flattened pedicles from T5 to T7 in keeping with an intraspinal mass.
Arif et al., ${ }^{1}$ Suslu et al. ${ }^{4}$ and Benzagmout et al. ${ }^{5}$ cite Braithwaite and Lees's classification of five types of spinal involvement in hydatid disease, of which the first three are rare:

1. Primary intramedullary hydatid cyst

2. Intradural extramedullary hydatid cyst

3. Extradural intraspinal hydatid cyst

4. Hydatid disease of the vertebrae

5. Paravertebral hydatid disease.

Our case falls into the third category. Spinal involvement is believed to occur through vertebral portovenous anastomosis. ${ }^{1,3,4,8}$

The hydatid cyst wall has three layers. The daughter cysts arise from the inner germinal layer; they eventually detach and float freely in the hydatid cyst fluid. The number of scolices increases within the daughter cysts, causing the cyst to enlarge. Symptoms are usually caused by the mass effect of the cyst.

Radiological examinations may be helpful in diagnosis. Plain $\mathrm{x}$-rays may show peripherally calcified lesions in the liver, spleen and other organs. ${ }^{1} \mathrm{CT}$ and MRI are the investigations of choice. The radiographic findings may be similar to those indicating tumours and infection, which makes differential diagnosis difficult. MRI is the best modality, as it shows the relationship to bony structures, the spinal cord and extension into soft tissues. ${ }^{2,6,8}$ The appearance of a hydatid cyst on MRI is quite characteristic: dome-shaped ends, with no debris in the lumen. ${ }^{6}$ The contents of the cyst have signal intensity similar to cerebrospinal fluid: low on T1W and high on T2W. The wall is sharply defined and hypointense on T1W images, which may show mild enhancement with intravenous

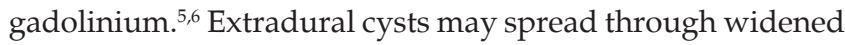
neural foramina into the muscle planes, as in our case.

The invasive nature of the disease in the spine makes total removal and therefore permanent eradication difficult. A recurrence risk of $30 \%-40 \%$ has been described. ${ }^{2}$
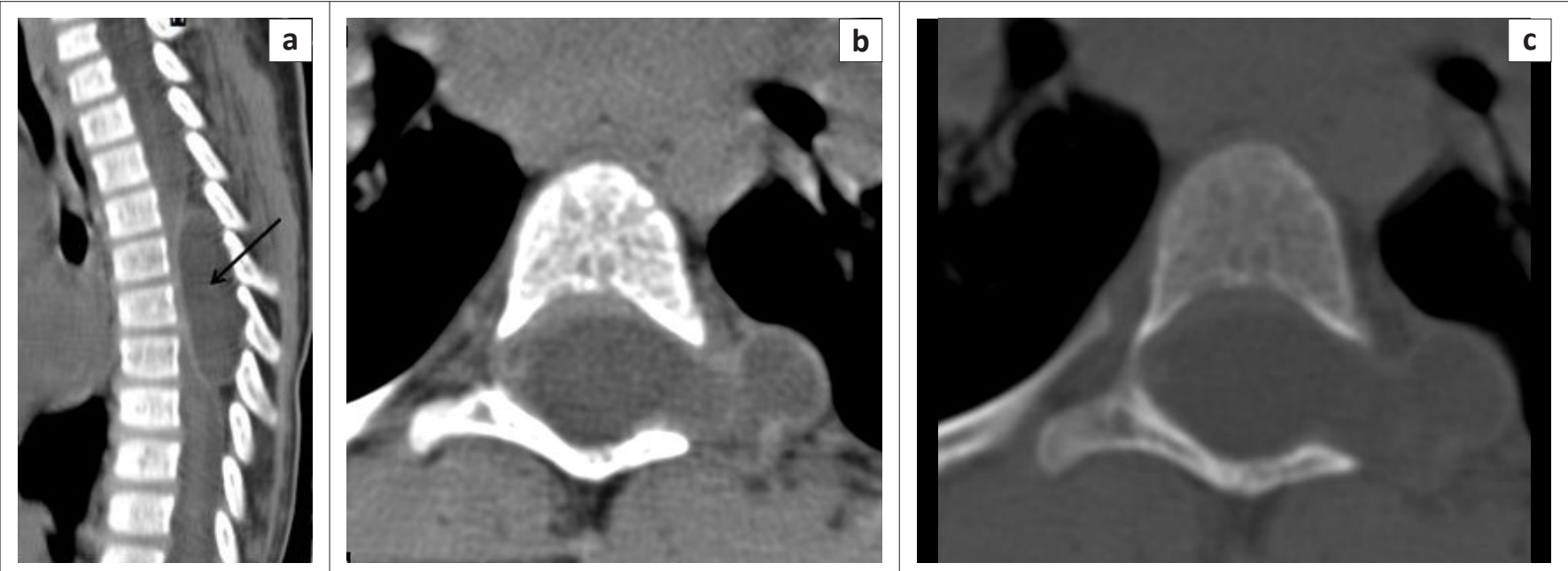

$\mathrm{CT}$, computed tomography.

FIGURE 2: (a) CT scan of the thoracic spine sagittal view on bone window demonstrates a widened canal with oval-shaped extradural cystic mass; (b) Axial CT scan soft tissue window demonstrates cystic mass extending through left $\mathrm{T} 7 / 6$ intervertebral foramen; (c) same level as (b), bone window demonstrates enlarged exit foramen at that level. 

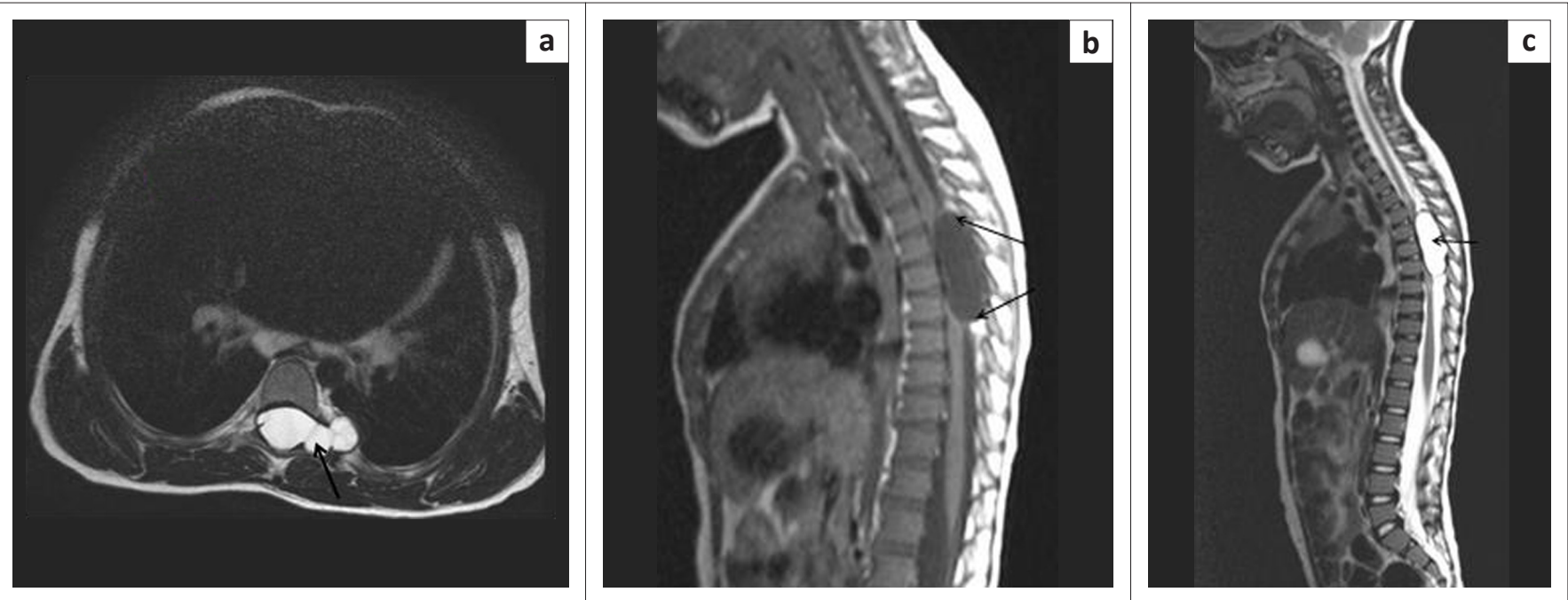

MRI, magnetic resonance imaging.

FIGURE 3: (a) Axial MRI showing T2 weighted image cyst (arrow) extending through exit foramen of T6-7; (b) Sagittal T1 weighted MRI image demonstrates the cyst to have dome-shaped ends (arrow) filling up the entire spinal canal with compression of cord from T4 to T7; (c) Sagital T2 weighted MRI image demonstrating cyst to be of high signal intensity with no solid components (arrow).

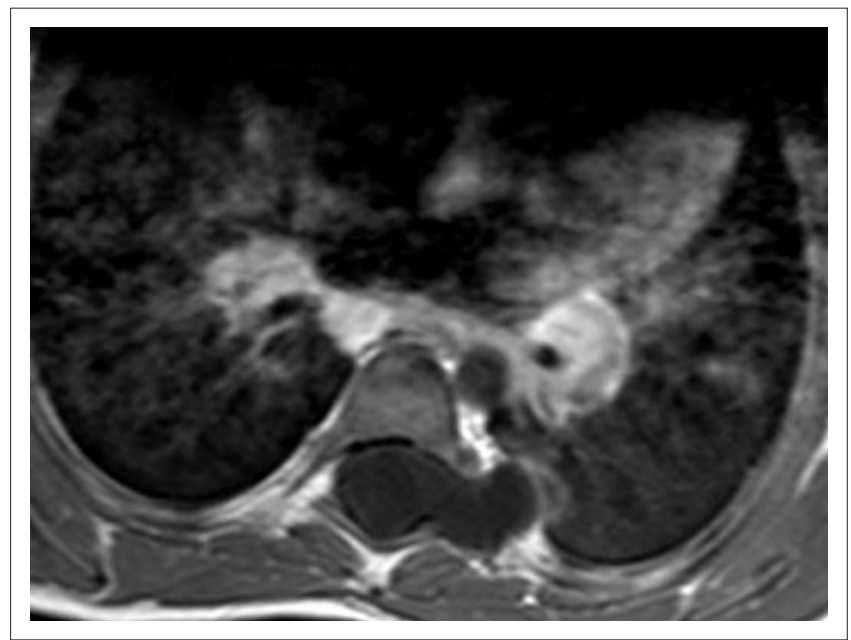

MRI, magnetic resonance imaging.

FIGURE 4: Axial gadolinium-enhanced MRI image does not demonstrate significant enhancement.

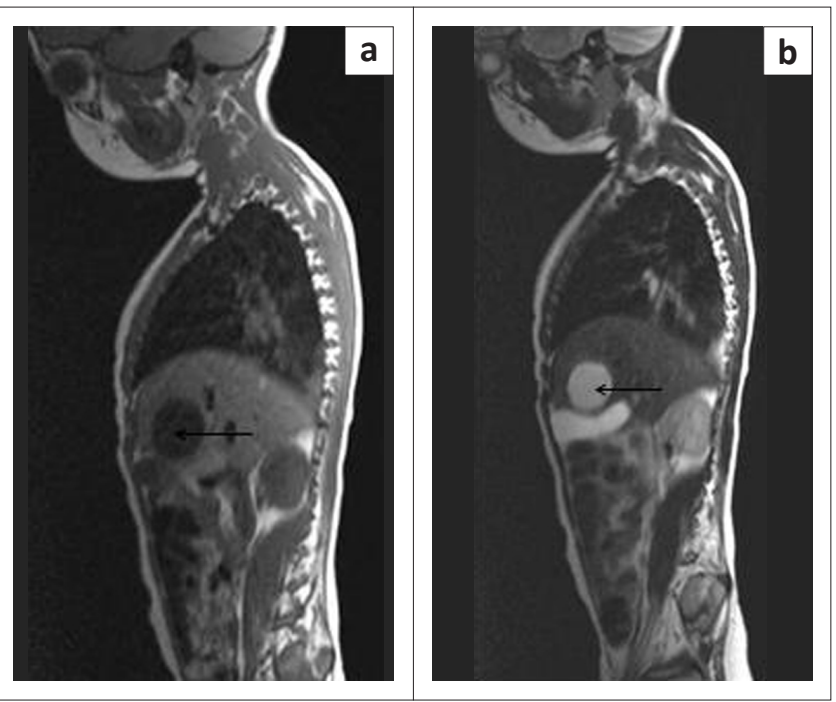

MRI, magnetic resonance imaging.

FIGURE 5: (a) and (b) Sagittal T1 and T2 weighted MRI images showing cystic lesion in segment 4 of the liver (arrows).

\section{Conclusion}

Hydatid disease should be included in the differential diagnosis in cases of cystic spinal cord masses causing paraplegia in endemic areas. Our case demonstrates a liver and extradural lesion without involvement of the vertebral column, confirming that the disease most likely spread via the portovenous route.

\section{Acknowledgements Competing interests}

The authors declare that they have no financial or personal relationship(s) that may have inappropriately influenced them in writing this article.

\section{Authors' contributions}

N.K. (Kalafong Hospital and University of Pretoria) was the project leader, whilst I.V. (Kalafong Hospital and University of Pretoria) did the literature search and editing.

\section{References}

1. Arif $\mathrm{SH}$, Zaheer $\mathrm{S}$. Intradural extramedullary primary hydatid cyst of the spine in a child: A very rare presentation. Eur Spine J. 2009;18(suppl 2):179-182. http:// dx.doi.org/10.1007/s00586-008-0777-8

2. Sapkas GS, Machinis TG, Chloros GD, et al. Spinal hydatid disease - A rare but existent pathological entity: Case report and review of the literature. South Med J. 2006;99(2):178-182. http://dx.doi.org/10.1097/01.smj.0000199747.81684.54

3. Scarlata F, Giordano S, Saporito L, et al. Cystic hydatidosis: A rare case of spine localization. Infez Med. 2011;1:39-41.

4. Suslu HT, Cecen A, Karaaslan A, et al. Primary spinal hydatid disease. Turk Neurosurg. 2009;19(2):186-188.

5. Benzagmout M, Kamaoui I, Chakour K, et al. Primary spinal epidural hydatid cyst with intrathoracic extension. Neurosciences. 2009;14(1):81-83.

6. Kahilogullari G, Tuna $\mathrm{H}$, Aydin Z, et al. Primary intradural extramedullary hydatid cyst. Am J Med Sci. 2005;329(4):202-204. http://dx.doi.org/10.1097/00000441 200504000-00006

7. Bhake A, Agrawal A. Hydatid disease of the spine. J Neurosci Rural Pract. 2010;1(2):61-62.

8. Gunecs $M$, Akdemir H, Tugcu B, et al. Multiple intradural spinal hydatid disease: A case report and review of literature. Spine. 2009;34(9):346-350. http://dx.doi. org/10.1097/BRS.0b013e3181a01b0 